\title{
Reliability and Sensitivity Analysis of a Batch Arrival Retrial Queue with k-Phase Services, Feedback, Vacation, Delay, Repair and Admission
}

\author{
S.Abdollahi ${ }^{1 *}$, M. R. Salehi Rad ${ }^{1}$ \\ Department of Statistics, Faculty of Statistics, Mathematics and Computer, Allameh Tabataba'i University, \\ Tehran, Iran
}

\begin{abstract}
Queueing theory is a way for real-world problems modeling and analyzing. In many processes, the input is converted to the desired output after several successive steps. But usually limitations and conditions such as Lack of space, feedback, vacation, failure, repair, etc. have a great impact on process efficiency.This article deals with the modeling the steady-state behavior of $\mathrm{a} M^{X} / G$ / 1retrial queueing system with $k$ phases of service. The arriving batches join the system with dependent admission due to the server state.If the customers find the server busy, they join the orbit to repeat their request. Although, the first phase of service is essential for all customers, any customer has three options after the completion of the $i-\operatorname{thphase}(i=1,2, \ldots, \mathrm{k})$. They may take the $(i+1)-t h$ phase of service with probability $\theta_{\mathrm{i}}$, otherwise return the orbit with probability $\mathrm{p}_{\mathrm{i}}$ or leave the system with probability $\left(1-p_{i}-\theta_{i}\right)$. Also, after each phase, the probabilistic failure, delay, repair and vacation are considered.In this article, after finding the steady-state distributions, the probability generating functions of the system and orbit size have been found. Then, some important performance measures of the system have been derived. Also, the system reliability has been defined. Eventually, to demonstrate the capability of the proposed model, the sensitivity analysis of performance measures via some model parameters (arrival/retrial/vacation rate) in different reliability levels have been investigated in a specific case of this model. Additionally, for optimizing the performance of system, some technical suggestions are presented.
\end{abstract}

Keyword:Bernoulli vacation, Feedback, Performance measures, Retrial queue, State-dependent admission, Repair, Delay, Reliability

\section{Introduction}

One way to identify the behavior of the systems in order to control and increase their productivity is to determine the model that they follow. On the other hand, without considering the priorities, real conditions and possible limitations for a system, the model fitting will not have the necessary efficiency.Today, increasing satisfaction of the customers is one of the most important priorities of dynamic systems. Sometimes, the customers arrive to the systems individually and sometimes in batches to receive services. For example, the sent products to the inspection test unit can be mentioned. In many systems, such as production of lines, achieving the desired result occurs after a multi-steps process. On the other hand, any system faces some limitations. One of these limitations is the lack of space for customers. In this case, the server is not ready to serve the customer at the

\footnotetext{
* Corresponding Author Email: abdollahi6028@yahoo.com
}

moment of arrival. Therefore, considering another space (called the orbit) for new customers to repeat their requests from there is one solution for this problem. This is known as the retrial phenomenon.Another constraint islimited resources and facilities. These systems have to impose restrictions on customer admission according to their conditionsdue to the state of the server (idle/busy). Also, dissatisfaction with the results of each step leads to incomplete process for reasons such as returning the customer to orbit for reservice or leaving the system. Sometimes the system has to be refreshed by going on a vacation. On the other hand, system failure, especially when it is not possible to repair the system immediately, and the increasing cost and time can't be neglected.Since reducing time and cost is one of the most important factors in customer satisfaction, overcoming these limitations is essential for the survival of the system. One of the useful techniques for modeling the systems and determining its performance measures (such as the mean of customers in the system and orbit and their waiting times) is the 
queueing theory. On the other hand, the reliability assessment is an effective approach to maintain and enhance the quality of the process output, increasing customer satisfaction and market share in the competitive world today. So far, many studies have been done by scientists in this regard. Some of them are as follows:

The optimizing of the phoneconversations in a call center by Erlang [1] was the first experience of using the queueing theory.

Afterward, the retrial queueing models have been investigated by several researchers such as Falin and Templeton [2]. Also, a literature of the investigations about the retrial queues has been presented by Artalejo [3].

Besides, the batch arrival of the customers has been studied by many researchers. Falin [4], Kulkarni [5] and Yamamuro [6] are some examples of this subject.

Some of the studied retrial queueing models have several essential or optional phases of services. Some of the works in this area have been done in this area are Kumar et al. [7], Choudhury and Deka [8], Wang and Li [9], Maurya [10], Jeganathan et al. [11], Rao et al. [12].

Also, returning to the orbit (feedback) is considered in many of the studied systems. For example, Kumar et al. [16], Choudhury and Paul [13], Arivudainambi and Godhandaraman [14], BadamchiZadeh [15], Som and Seth [16], Rajadurai et al. [17], Bouchentouf et al. [18] have considered the feedback assumption in their models.

On the other hand, depending on the situation, different systems face different types of vacations, such as general vacation by Senthikumar and Arumuganathan[19], modified vacation by Jain and Bhagat[20], Bernoulli vacation by Choudhury and $\mathrm{Ke}[21]$, working vacation by Azhagappan[22] and variant vacation by $\mathrm{Ke}[23]$.

Occurrence of the breakdown/failure isthe inevitable issue for any system. Therefore, in designing any system, preventive or corrective actions should be planned. So, thebreakdowns/failures repairis one of the most important topics in this program. Therefore, this issue has been considered in many of the studied systems such as V. G. Kulkarni and Bong Dae Choi [24] and P.Rajaduraia et al. [25].

But sometimes due to some limitations, these repairs are delayed.Madhu Jain and AmitaBhagat [26] and Choudhury and $\mathrm{Ke}[21]$ have been considered this issue in their model.

Some systems aren't able to respond to all customers. So, they have to impose restrictions on customer admission according to their conditions due to the state of the server (idle/busy). In this relation, Choudhury and Deka [27-28] have considered the Bernoulli admission mechanism in their model.

Improving the system reliability is one way to achieve the secure system. The reliability of multi- component systems was studied by Birnbaun et al. [29]. Also, these subjects have been considered in the queueing models by some authors such as Li et al. [30], Tang [31], Wang et al. [32] and Achcar and Piratelli[33].

In this article, modeling and analyzing a $M^{X} / G /$ 1retrial queue system with k-phases of heterogeneous services in succession with first essential and $k-$ 1 optional phases, and state-dependent admission have been studied. Also, after each phase, the probabilistic feedback, failure, delay, repair, and vacation have been considered. Also, by considering the successful delivery of all service stages as the system successful, the conception of reliability has been defined and the reliability analysis hasbeen done. Of course, there exist the other definitions of the concept of reliability for other models which can be referred to [34-36].

Despite many valuable studies, any system has not been studied with these conditions. The novelties of this article are considering all of the above conditions in a system together, modeling and obtaining the performance measures of the system and reliability and sensitivity analysis of a special case of it. In this relation, the queueingmethod for modeling and analysis of systems with process approaches hasbeen considered. This model is applicable in many processes such as telecommunication systems, telephone switching systems, computer networks, and inspection tests of products.

For this model, the steady-state distributions, the probability generating functions of the system and orbit size have been found. Then, the performance measures have been obtained by using the supplementary variable technique.

In summarizing, the main contributions of this article are as below:

1) Considering batch arrival, state-dependent admission and (after each phase) the probabilistic feedback, failure, delay, repair, and vacation conditions together in a k-phases retrial queueing system with first essential and $k-1$ optional phases,

2) Having three choices for customers after $i-$ thphase $(i=1,2, \ldots, \mathrm{k}-1)$

i. going to $(i+1)-$ thphase service with probability $\theta_{i}\left(\theta_{k}=0\right)$,

ii. going to orbit with probability $p_{i}$,

iii. leaving the system with probability $\left(1-p_{i}-\theta_{i}\right)$,

3) Considering general assumptions such as arbitrary distributions of retrial/service times, different probabilities at each phase, and variable size of arrival batches to have a comprehensive model to contain different systems in special cases,

4) Considering the system reliability and its effect in sensitivity analysis, 
5) Providing an applicable example in the engineering field with technical suggestions,

6) Using the queueing method for modeling and analysis of the systems with process approach.

This paper is organized as follows. The model description is given in section 2. Section 3 deals with the analysis of the system containing the definitions, steady-state equations, and $\mathrm{PGF}^{\dagger} \mathrm{s}$. The PGFs of the system and orbit size and some important performance measures are obtained in section 4. Eventually, in section 5 , by some numerical examples, the sensitivity of some performance measures isinvestigated. Also, the conclusions are provided in the section 6 .

\section{Model Description}

The considered retrial queue has the following assumptions:

A. The customers arrive in batches from outside the system according to a Poisson process with arrival rate $\lambda$ with admission depending to the state of server. So, the probabilities of arrival $\operatorname{are} \alpha_{1}$ when the server is idle, $\alpha_{2}$ when the server is busy, $\alpha_{3}$ when the server is on vacation, $\alpha_{4}$ when the server is in delay and $\alpha_{5}$ when the server is under repair. The probability mass function (p.m.f) of the size of batches is $c_{m}=\operatorname{Pr}(X=m) ; m \geq 1, \quad$ withPGF $C(z)=E\left[z^{X}\right]$ and the first two factorial moments $C_{[1]}$ and $C_{[2]}$ are finite.

B. There is no waiting space and if the server is busy, the arriving batches enter a retrial group (orbit) to repeat the request for service with the FCFS discipline. Otherwise, one customer of the arriving batch takes the service and the others enter the orbit.

C. If an arriving customer (primary or retrial) finds the server idle, then he/she/it enters immediately to take the first phase of service. The concept of the primary customer is the customer who has arrived to the system for the first time.

D. The retrial times are generally distributed with distribution function $A(x)$, density function $a(x)$ and Laplace transform $A^{*}(\theta)$. Also, the first and second moments of this distribution are finite.

E. The server provides $k$ phases of heterogeneous services in succession. The first phase is essential for all customers. At the end of $i-t h$ phase of service $(i=1,2, \ldots, k-1)$, the customer may take the $(i+1)-t h$ phase with probability $\theta_{i}$, return to orbit to repeat the retrial to take the service again with probability $\mathrm{p}_{\mathrm{i}}$ or depart the system with probability $1-\theta_{\mathrm{i}}-\mathrm{p}_{\mathrm{i}}$. The service times are independent and for $i-t h$ phase denoted by the random variable $B_{i}$ with general distribution functions $B_{i}(x)$, density functions $b_{i}(x)$ and Laplace transforms $B_{i}^{*}(\theta)(i=1,2, \ldots, k)$. Also, the first and second moments of these distributions are finite.

F. At the end of $i-t h$ phase $(i=1,2, \ldots, k)$, if the applicant does not go to $(i+1)-t h$ phase the server can have a vacation with probability $\tau_{\mathrm{i}}$ or may continue the new service with probability $1-\tau_{\mathrm{i}}$. Vacation times are random variables with general distribution function $V_{i}(x)$, density function $\mathrm{v}_{i}(x)$ and Laplace transform $V_{i}^{*}(\theta)$. The first and second moments of this distribution are finite.

G. The server may fail at any phase. The life of the equipment used for the $i-t h$ phase service has an exponential distribution with parameter $1 / a_{\mathrm{i}},(i=1,2, \ldots, k)$.

H. The delay time that occurred from the time of failure of the system to the time of repair at $i-t h$ phase $(i=1,2, \ldots, k)$ has a random variable $D_{\mathrm{i}}$ with general distribution functions $D_{i}(x)$, density functions $d_{i}(x)$ and Laplace transforms $D_{i}^{*}(\theta)(i=1,2, \ldots, k)$. Also the first and second moments of these distributions are finite.

I. The repair time of the system faced with failure in the $i-t h$ phase has a random variable $R_{\mathrm{i}}$ with general distribution functions $R_{i}(x)$, density functions $r_{i}(x)$ and Laplace transforms $R_{i}^{*}(\theta)(i=1,2, \ldots, k)$. Also the first and second moments of these distributions are finite.

\section{Analysis of the model}

To analyze the model, first, the state of the system is recognized. Since the distribution of the service times is unknown (general), thus, this model doesn't hasthe Markovian property. But, an embedded Markov chain can be defined.

For this, the state of the system at time $t$ by the Markov process $Z(t)=\{N(t), X(t)\}$ is considered, in which for $1 \leq i \leq k$ :

$$
J(t)=\left\{\begin{array}{rr}
1 & \text { the server is idle } \\
2 & \text { the server is busy at phase } i \\
3 & \text { the server ison vacation at phase } i \\
4 & \text { the server is in delay at phase } i \\
5 & \text { the server is under repair at phase } i
\end{array}\right.
$$

\footnotetext{
${ }^{\dagger}$ Probability generating functions
} 
$X(t)= \begin{cases}A^{0}(t) & J(t)=1 \\ B_{i}^{0}(t) & J(t)=2 \\ V_{i}^{0}(t) & J(t)=3 \\ D_{i}^{0}(t) & J(t)=4 \\ R_{i}^{0}(t) & J(t)=5\end{cases}$

Also, $N(t)$ corresponds the number of customers in the retrial queue at time $t$.

Definition 1 For $i=1,2, \ldots, k$,

a) The service conditional completion rates at time $x$ is

$$
\mu_{i}(x)=b_{i}(x) /\left(1-B_{i}(x)\right)
$$

by assumptions $B_{i}(0)=0, \quad B_{i}(\infty)=1$ and that $B_{i}(x)(i=1,2, \ldots, k)$ are continuous at $x=0$.

b) The vacation conditional completion rates at time $x$ is

$$
v_{i}(x)=\mathrm{v}_{i}(x) /\left(1-V_{i}(x)\right)
$$

by assumptions $V_{i}(0)=0, V_{i}(\infty)=1$ and that $V_{i}(x)(i=1,2, \ldots, k)$ are continuous at $x=0$.

c) The delay conditional completion rates at time $x$ is

$$
\gamma_{i}(y)=\mathrm{d}_{i}(y) /\left(1-D_{i}(y)\right)
$$

by assumptions $D_{i}(0)=0, D_{i}(\infty)=1$ and that $D_{i}(y)(i=1,2, \ldots, k)$ are continuous at $\mathrm{y}=0$.

d) The repair conditional completion rates at time $x$ is

$$
\varepsilon_{i}(y)=g_{i}(y) /\left(1-G_{i}(y)\right)
$$

by assumptions $G_{i}(0)=0, G_{i}(\infty)=1$ and that $G_{i}(y)(i=1,2, \ldots, k)$ are continuous at $\mathrm{y}=0$.

e) The repeated attempts (retrial) rate at time $x$ is

$$
\begin{aligned}
& \qquad \eta(x)=a(x) /(1-A(x)) \\
& \text { where } A(0)=0, \quad A(\infty)=1 \text { and } A(x) \text { is } \\
& \text { continuous at } x=0 .
\end{aligned}
$$

Definition2The reliability function of the system/applicants is defined as:

$R E=q_{k} \cdot R E_{k-1}$

where

$$
\begin{aligned}
& R E_{i}=\prod_{j=1}^{i} \theta_{j} \quad i=1,2, \ldots k \quad ; \quad R E_{0}=\theta_{0}=1 \\
& ; \quad R E_{k}=0 .
\end{aligned}
$$

According to the stated points in this section, the theory of the model can be extended in the following.
First, the stationary distribution of the Markovprocess $\{Z(t) ; t \geq 0\} \quad$ under the stability condition is found. For this goal, the bellow probabilities are defined:

$I_{0}(t)=p\left\{X(t)=A^{0}(t), N(t)=0\right\}$

$\lim _{t \rightarrow \infty} I_{0}(t)=I_{0}$

where $J(t)$ and $N(t)$ have been defined before and the probability densities are:

$I_{n}(t, x)=p\left\{X(t)=A^{0}(t), N(t)=n, x \leq\right.$

$\left.A^{0}(t)<x+d x\right\}, t \geq 0, x \geq 0, n \geq 1$

$\lim _{t \rightarrow \infty} I_{n}(t, x)=I_{n}(x), \quad x \geq 0, n \geq 1$

and for $i=1, \ldots, k$,

$\begin{aligned} P_{i, n}(t, x)=p\{X(t) & =B_{i}^{0}(t), N(t)=n, x \leq B_{i}^{0}(t) \\ & <x+d x \quad\}, \quad t \geq 0, x \geq 0, n \\ \geq & 0\end{aligned}$

$\lim _{t \rightarrow \infty} P_{i, n}(t, x)=P_{n}(x), x \geq 0, n \geq 0, i=1,2, \ldots, k$

$V_{i, n}(t, x)=p\left\{X(t)=V_{i}^{0}(t), N(t)=n, x \leq V_{i}^{0}(t)\right.$

$<x+d x\}$,

$t \geq 0, x \geq 0, n \geq 0$

$\lim _{t \rightarrow \infty} V_{i, n}(t, x)=V_{i, n}(x), x \geq 0, n \geq 0$

$D_{i, n}(t, x, y)$

$=p\left\{X(t)=D_{i}^{0}(t), N(t)=n, y \leq D_{i}^{0}(t)<y+d y \mid B_{i}^{(}\right.$

$\geq 0, y \geq 0, x \geq 0, n \geq 0$

$\lim _{t \rightarrow \infty} D_{i, n}(t, x, y)=D_{i, n}(x, y), y \geq 0, x \geq 0, n \geq 0$

$D_{i, n}(t, x, y)=$

$p\left\{X(t)=D_{i}^{0}(t), N(t)=n, y \leq D_{i}^{0}(t)<y+d y \mid B_{i}^{0}(t)=x\right\}$

$$
t \geq 0, y \geq 0, x \geq 0, n \geq 0
$$

$\lim _{t \rightarrow \infty} R_{i, n}(t, x, y)=R_{i, n}(x, y), y \geq 0, x \geq 0, n \geq 0$

Now, by using the supplementary variable technique the steady state equations can be obtained for $i=1, \ldots, k$ as follow: 


$$
\begin{aligned}
\lambda I_{0}=\sum_{i=1}^{k-1}\left(1-\theta_{i}\right. & \left.-p_{i}\right)(1 \\
& \left.-\tau_{i}\right) \int_{0}^{\infty} \mu_{i}(x) P_{i, 0}(x) d x \\
& +\int_{0}^{\infty} v_{i}(x) V_{i, 0}(x) d x
\end{aligned}
$$

$\left(d I \_n(x)\right) / d x+(\lambda+\eta(x)) I \_n(x)$

$=\lambda\left(1-\alpha \_1\right) I n(x)$

$n \geq 1$

$$
\begin{aligned}
d P_{i, 0}(x) / d x+(\lambda & \left.+\mu_{i}(x)+a_{i}\right) P_{i, 0}(x) \\
& =\lambda\left(1-\alpha_{2}\right) P_{i, 0}(x) \\
& +\int_{0}^{\infty} \varepsilon_{i}(y) R_{i, 0}(x, y) d y
\end{aligned}
$$$$
d P_{i, n}(x) / d x+\left(\lambda+\mu_{i}(x)+a_{i}\right) P_{i, n}(x)
$$$$
=\lambda\left(1-\alpha_{2}\right) P_{i, n}(x)+\lambda \alpha_{2} \sum_{m=1}^{n} c_{m} P_{i, n-m}(x)
$$$$
+\int_{0}^{\infty} \varepsilon_{i}(y) R_{i, n}(x, y) d y \quad n \geq 1
$$

$$
\begin{aligned}
& d D_{i, n}(x, y) / d x+\left(\lambda+\gamma_{i}(y)\right) D_{i, n}(x, y) \\
& =\lambda\left(1-\alpha_{4}\right) D_{i, n}(x, y) \\
& +\lambda \alpha_{4} \sum_{m=1}^{n} c_{m} D_{i, n-m}(x, y) \quad n \geq 1
\end{aligned}
$$

$$
\begin{aligned}
d R_{i, 0}(x, y) / d y+ & \left(\lambda+\varepsilon_{i}(y)\right) R_{i, 0}(x, y) \\
& =\lambda\left(1-\alpha_{5}\right) R_{i, 0}(x, y)
\end{aligned}
$$

$d R_{i, n}(x, y) / d x+\left(\lambda+\varepsilon_{i}(y)\right) R_{i, n}(x, y)$

$=\lambda\left(1-\alpha_{5}\right) R_{i, n}(x, y)$

$+\lambda \alpha_{5} \sum_{m=1}^{n} c_{m} R_{i, n-m}(x, y) \quad n \geq 1$

in which for $i=1,2, \ldots, k$, the boundary conditions are as below:

$$
\begin{aligned}
& I_{n}(0)=\sum_{i=1}^{k}\left(1-\tau_{i}\right) p_{i} \int_{0}^{\infty} \mu_{i}(x) P_{i, n-1}(x) d x \\
& +\sum_{i=1}^{k}\left(1-\tau_{i}\right)\left(1-\theta_{i}-p_{i}\right) \int_{0}^{\infty} \mu_{i}(x) P_{i, n}(x) d x \\
& +\sum_{i=1}^{k} \int_{0}^{\infty} v_{i}(x) V_{i, n}(x) d x, n \\
& \geq 1 \\
& P_{1,0}(0)=\lambda c_{1} I_{0}+\int_{0}^{\infty} I_{1}(x) \eta(x) d x,
\end{aligned}
$$

$P_{1, n}(0)$

$=\lambda c_{n+1} I_{0}+\lambda \alpha_{1} \sum_{m=1}^{n} c_{m} \int_{0}^{\infty} I_{n-m+1}(x) d x$

$+\int_{0}^{\infty} \eta(x) I_{n+1}(x) d x, \quad n \geq 1$

$$
\begin{gathered}
P_{i, n}(0)=\theta_{i-1} \int_{0}^{\infty} P_{i-1, n}(x) \mu_{i-1}(x) d x, \\
n \geq 1 \quad, i=2, \ldots, k \\
V_{i, 0}(0) \\
=\tau_{i}\left(1-\theta_{i}-p_{i}\right) \int_{0}^{\infty} \mu_{i}(x) P_{i, 0}(x) d x, \\
V_{i, n}(0)=\tau_{i} p_{i} \int_{0}^{\infty} P_{i, n-1}(x) \mu_{i}(x) d x \\
\quad+\int_{0}^{\infty} \tau_{i}\left(1-\theta_{i}\right. \\
\left.\quad-p_{i}\right) P_{i, n}(x) \mu_{i}(x) d x \\
\geq 1
\end{gathered}
$$

$D_{i, n}(x, 0)=a_{i} P_{i, n}(x)$

$R_{i, n}(x, 0)=\int_{0}^{\infty} \gamma_{i}(y) D_{i, n}(x, y) d y$

and the normalized condition is 


$$
\begin{aligned}
I_{0}+\sum_{n=1}^{\infty} \int_{0}^{\infty} I_{n}(x) & d x \\
& +\sum_{n=0}^{\infty} \sum_{i=1}^{k}\left\{\int_{0}^{\infty} P_{i, n}(x) d x\right. \\
& +\int_{0}^{\infty} V_{i, n}(x) d x \\
& +\int_{0}^{\infty} \int_{0}^{\infty} D_{i, n}(x) d x d y \\
& \left.+\int_{0}^{\infty} \int_{0}^{\infty} R_{i, n}(x) d x d y\right\}
\end{aligned}
$$

Now, the PGFs are defined as below:

$$
\begin{aligned}
I(x, z) & =\sum_{n=1}^{\infty} I_{n}(x) z^{n}, \\
I(0, z) & =\sum_{n=1}^{\infty} I_{n}(0) z^{n}, \\
P_{i}(x, z) & =\sum_{n=0}^{\infty} P_{i, n}(x) z^{n}, \\
i & =1,2, \ldots ., k \\
P_{i}(0, z) & =\sum_{n=0}^{\infty} P_{i, n}(0) z^{n}, \\
i & =1,2, \ldots ., k
\end{aligned}
$$$$
V_{i}(x, z)=\sum_{n=0}^{\infty} V_{i, n}(x) z^{n},
$$$$
i=1,2, \ldots ., k
$$$$
V_{i}(0, z)=\sum_{n=0}^{\infty} V_{i, n}(0) z^{n},
$$$$
i=1,2, \ldots \ldots, k
$$$$
D_{i}(x, y, z)=\sum_{n=0}^{\infty} D_{i, n}(x, y) z^{n},
$$$$
i=1,2, \ldots \ldots, k
$$$$
D_{i}(x, 0, z)=\sum_{n=0}^{\infty} D_{i, n}(x, 0) z^{n},
$$$$
i=1,2, \ldots \ldots, k
$$

$$
\begin{gathered}
R_{i}(x, y, z)=\sum_{n=0}^{\infty} R_{i, n}(x, y) z^{n}, \\
i=1,2, \ldots \ldots, k
\end{gathered}
$$

$$
\begin{gathered}
R_{i}(x, 0, z)=\sum_{\substack{n=0 \\
i}}^{\infty} R_{i, n}(x, 0) z^{n}, \ldots, k \\
\text {, } 2, \ldots . .,
\end{gathered}
$$

By multiplying equations (16)-(24) by $z^{n}$ and summation on $n,(44)-(48)$ are obtained:

$$
\begin{aligned}
& \partial I(x, z) / \partial x+\left[\lambda \alpha_{1}+\eta(x)\right] I(x, z)=0 \\
& \partial P_{i}(x, z) / \partial x+\left[\lambda+\mu_{i}(x)+a_{i}\right] P_{i}(x, z) \\
& =\lambda\left(1-\alpha_{2}\right) P_{i}(x, z) \\
& +\lambda \alpha_{2} C(z) P_{i}(x, z) \\
& +\int_{0}^{\infty} \varepsilon_{i}(y) R_{i}(x, y, z) d y \\
& \partial V_{i}(x, z) / \partial x+\left[\lambda+v_{i}(x)\right] V_{i}(x, z) \\
& =\lambda\left(1-\alpha_{3}\right) V_{i}(x, z) \\
& +\lambda \alpha_{3} C(z) V_{i}(x, z) \\
& \begin{aligned}
\partial D_{i}(x, y, z) / \partial y+ & {\left[\lambda+\gamma_{i}(y)\right] D_{i}(x, y, z) } \\
& =\lambda\left(1-\alpha_{4}\right) D_{i}(x, y, z) \\
& +\lambda \alpha_{4} C(z) D_{i}(x, y, z)
\end{aligned} \\
& \partial R_{i}(x, y, z) / \partial y+\left[\lambda+\varepsilon_{i}(y)\right] R_{i}(x, y, z) \\
& =\lambda\left(1-\alpha_{5}\right) R_{i}(x, y, z) \\
& +\lambda \alpha_{5} C(z) R_{i}(x, y, z)
\end{aligned}
$$

Theorem 1The PGFs for $|z| \leq 1$ and $i=1,2, \ldots, k$ are:

$$
\begin{aligned}
& I(x, z)=I(0, z)[1-A(x)] \exp \left(-\lambda \alpha_{1} x\right) \\
& P_{i}(x, z)=P_{i}(0, z)\left[1-B_{i}(x)\right] \exp \left(-x h_{i}(z)\right) \\
& \begin{aligned}
h_{i}(z)=\lambda \alpha_{2}(1- & C(z)) \\
& +a_{i}[1 \\
& -D_{i}^{*}\left(\lambda \alpha_{4}(1\right. \\
& -C(z)))] G_{i}^{*}\left(\lambda \alpha_{5}(1\right. \\
- & C(z)))
\end{aligned}
\end{aligned}
$$

$$
\begin{gathered}
P_{i}(x, z)=P_{i}(0, z)\left[1-B_{i}(x)\right] \exp \left(-x h_{i}(z)\right) \\
D_{i}(x, y, z)=D_{i}(x, 0, z)[1 \\
\left.-D_{i}(y)\right] \exp \left(-\lambda \alpha_{4}(1\right. \\
-C(z)) y)
\end{gathered}
$$

$$
\begin{aligned}
R_{i}(x, y, z)=R_{i}( & x, 0, z)[1 \\
& \left.-G_{i}(y)\right] \exp \left(-\lambda \alpha_{5}(1\right. \\
& -C(z)) y)
\end{aligned}
$$


where

$$
\begin{aligned}
& D_{i}^{*}\left(\lambda \alpha_{4}(1-C(z))\right) \\
& =\int_{0}^{\infty} \exp \left(-\lambda \alpha_{4}(1-C(z)) y\right) d D_{i}(y) \\
& G_{i}^{*}\left(\lambda \alpha_{4}(1-C(z))\right){ }^{\infty}=\int_{0}^{\infty} \exp \left(-\lambda \alpha_{5}(1-C(z)) y\right) d G_{i}(y)
\end{aligned}
$$

ProofBy solving the differential equations (44)-(48) the above relations can be obtained. For example, (50) has been obtained by using (45), as below:

$$
\begin{aligned}
& \frac{\partial / \partial x P_{i}(x, z)}{P_{i}(x, z)}=-\left[\mu_{i}(x)+a_{i}+\lambda \alpha_{2}+\lambda \alpha_{2} C(z)\right. \\
& \left.+\frac{\int_{0}^{\infty} \varepsilon_{i}(y) R_{i}(x, y, z) d y}{P_{i}(x, z)}\right] \\
& \int_{0}^{\infty} \varepsilon_{i}(y) R_{i}(x, y, z) d y \\
& =\int_{0}^{\infty} R_{i}(x, 0, z)[1 \\
& \left.-G_{i}(y)\right] \exp \left(-\lambda \alpha_{5}(1\right. \\
& -C(z)) y) \varepsilon_{i}(y) d y \\
& =R_{i}(x, 0, z) \int_{0}^{\infty} g_{i}(y) \exp \left(-\lambda \alpha_{5}(1-C(z)) y\right) d y \\
& =R_{i}(x, 0, z) G_{i}^{*}\left(\lambda \alpha_{5}(1-C(z))\right)
\end{aligned}
$$

also, by using (32):

$$
\begin{aligned}
& R_{i}(x, 0, z)=\int_{0}^{\infty} D_{i}(x, y, z) \gamma_{i}(y) d y \\
&=\int_{0}^{\infty} D_{i}(x, 0, z)[1 \\
&\left.-D_{i}(y)\right] \exp \left(-\lambda \alpha_{4}(1\right. \\
&-C(z)) y) \gamma_{i}(y) d y \\
&=D_{i}(x, 0, z) D_{i}^{*}\left(\lambda \alpha_{4}(1-C(z))\right)
\end{aligned}
$$

and by using (31):

$$
\begin{aligned}
& \int_{0}^{\infty} \frac{\int_{0}^{\infty} \varepsilon_{i}(y) R_{i}(x, y, z) d y}{P_{i}(x, z)} d x \\
& =\int_{0}^{\infty} \frac{a_{i} P_{i}(x, z) D_{i}^{*}\left(\lambda \alpha_{4}(1-C(z))\right) G_{i}^{*}\left(\lambda \alpha_{5}(1-C(z))\right)}{P_{i}(x, z)} d x \\
& =a_{i} D_{i}^{*}\left(\lambda \alpha_{4}(1-C(z))\right) G_{i}^{*}\left(\lambda \alpha_{5}(1-C(z))\right) x
\end{aligned}
$$

so,

$$
\begin{aligned}
& P_{i}(x, z) \\
& =e^{-\int_{0}^{\infty} \mu_{i}(x) d x} \exp \left\{\left[-a_{i}\right.\right. \\
& -\lambda \alpha_{2}(1 \\
& -C(z))] x\} \exp \left\{\int_{0}^{\infty} \frac{\int_{0}^{\infty} \varepsilon_{i}(y) R_{i}(x, y, z) d y}{P_{i}(x, z)} d x\right\}
\end{aligned}
$$

and also, by using the formula (28) and (37),

$P_{i}(0, z)=R_{i-1} X_{i-1}^{*} P_{1}(0, z)$

sothat

$$
X_{i}^{*}=\prod_{j=1}^{i} B_{j}^{*}\left(h_{j}(z)\right)
$$

and

$$
R_{i}=\prod_{j=1}^{i} \theta_{j}
$$

$$
\theta_{0}=1, \quad \theta_{k}=0, \quad R_{0}=1, \quad R_{k}=0
$$

In addition, $\quad P_{1}(0, z), \quad P_{i}(0, z), \quad I(0, z), \quad V_{i}(0, z)$, $D_{i}(x, 0, z)$ and $R_{i}(x, 0, z)$ have been obtained as bellow:

$$
\begin{gathered}
P_{1}(0, z)=\sum_{n=0}^{\infty} P_{1, n}(0) z^{n}=\lambda c_{1} I_{0}+\int_{0}^{\infty} I_{1}(x) \eta(x) d x+ \\
\sum_{n=1}^{\infty} P_{1, n}(0) z^{n}=\lambda I_{0} \sum_{n=0}^{\infty} C_{n+1} z^{n}+ \\
\int_{0}^{\infty} \sum_{n=0}^{\infty} I_{n+1}(x) z^{n} \eta(x) d x \\
+\lambda \alpha_{1} \sum_{n=0}^{\infty} \sum_{m=1}^{n} C_{m} \int_{0}^{\infty} z^{n} I_{n-m+1}(x) d x
\end{gathered}
$$

so,

$$
\begin{aligned}
& z P_{1}(0, z) \\
& =\lambda I_{0} \sum_{n=0}^{\infty} C_{n+1} z^{n+1}+\int_{0}^{\infty} \sum_{n=0}^{\infty} z^{n+1} I_{n+1}(x) \eta(x) d x \\
& +\lambda \alpha_{1} \sum_{m=1}^{n} C_{m} \int_{0}^{\infty} \sum_{n=1}^{\infty} z^{n+1} I_{n-m+1}(x) d x \\
& =\lambda I_{0} C(z)+\int_{0}^{\infty} I(x, z) \eta(x) d x \\
& +\lambda \alpha_{1} \sum_{n=0}^{\infty} \sum_{m=1}^{n} C_{m} z^{m} \int_{0}^{\infty} z^{n-m+1} I_{n-m+1}(x) d x \\
& =\lambda I_{0} C(z)+\int_{0}^{\infty} I(x, z) \eta(x) d x \\
& +\lambda \alpha_{1} C(z) \int_{0}^{\infty} I(x, z) d x
\end{aligned}
$$

Now, by using (49) the bellow relation have been obtained:

$$
=P_{i}(0, z)\left[1-B_{i}(x)\right] \exp \left(-x h_{i}(z)\right)
$$




$$
\begin{aligned}
& z P_{1}(0, z) \\
& =\lambda I_{0} C(z) \\
& +I(0, z) \int_{0}^{\infty}[1 \\
& \text { - } \quad A(x)] \exp \left(-\lambda \alpha_{1} x\right) \eta(x) d x \\
& +\quad \lambda \alpha_{1} C(z) I(0, z) \int_{0}^{\infty}[1 \\
& -\quad A(x)] \exp \left(-\lambda \alpha_{1} x\right) d x \\
& =\lambda I_{0} C(z)+I(0, z) A^{*}\left(\lambda \alpha_{1}\right) \\
& +\quad \lambda \alpha_{1} C(z) I(0, z) \int_{0}^{\infty}[1 \\
& \text { - } \quad A(x)] \exp \left(-\lambda \alpha_{1} x\right) d x \\
& =\lambda I_{0} C(z) \\
& +I(0, z)\left\{A^{*}\left(\lambda \alpha_{1}\right)\right. \\
& +\quad C(z)[1
\end{aligned}
$$

Also, by using (29), (30) and (55), $V_{i}(0, z)$ can be obtained:

$$
\begin{aligned}
& V_{i}(0, z)=\sum_{n=0}^{\infty} V_{i, n}(0) z^{n} \\
& =\int_{0}^{\infty} \tau_{i}\left[1-\theta_{i}\right. \\
& -p_{i}(1 \\
& -z)] P_{i}(x, z) \mu_{i}(x) d x \\
& =\tau_{i}\left[1-\theta_{i}-p_{i}(1-z)\right] P_{i}(0, z) B_{i}^{*}\left(h_{i}(z)\right) \\
& =\tau_{i}\left[1-\theta_{i}-p_{i}(1-z)\right] P_{1}(0, z) R_{i-1} X_{i}^{*}
\end{aligned}
$$

And by using (25) and (35):

$I(0, z)=\sum_{i=1}^{k}\left(1-\tau_{i}\right) p_{i} z \int_{0}^{\infty} \mu_{i}(x) P_{i}(x, z) d x+$ $\sum_{i=1}^{k}\left(1-\tau_{i}\right)\left(1-\theta_{i}-p_{i}\right) \int_{0}^{\infty} \mu_{i}(x) P_{i}(x, z) d x+$ $\sum_{i=1}^{k} \int_{0}^{\infty} v_{i}(x) V_{i}(x, z) d x-\lambda I_{0}$

And by using (55) and (57):

$$
\begin{gathered}
I(0, z)=P_{1}(0, z) \sum_{i=1}^{k}\left\{\left(1-\tau_{i}\right) p_{i} z+\left(1-\tau_{i}\right)(1-\right. \\
\left.\left.\theta_{i}-p_{i}\right)+\tau_{i}\left[1-\theta_{i}-p_{i}(1-z)\right]\right\} R_{i-1} X_{i}^{*}-\lambda I_{0} \\
=P_{1}(0, z) F(z)-\lambda I_{0}
\end{gathered}
$$

Now, by substituting $P_{1}(0, z)$ from $(56), I(0, z)$ can be obtained:

$$
I(0, z)=\frac{\lambda I_{0}[C(z) F(z)-z]}{z-F(z)\left[A^{*}\left(\lambda \alpha_{1}\right)+C(z)\left(1-A^{*}\left(\lambda \alpha_{1}\right)\right)\right]}
$$

In witch,

$$
\begin{array}{r}
F(z)=\sum_{i=1}^{k}\left\{\left(1-\tau_{i}\right) p_{i} z+\left(1-\tau_{i}\right)\left(1-\theta_{i}-p_{i}\right)\right. \\
\left.+\tau_{i}\left[1-\theta_{i}-p_{i}(1-z)\right]\right\} R_{i-1} X_{i}^{*}
\end{array}
$$

Obtaining relations $R_{i}(x, 0, z)$ and $D_{i}(x, 0, z)$ has already been discussed before.

\section{Theorem 2}

Under the steady state condition, the joint distributions of the server state have the following partial generating functions:

$$
\begin{aligned}
& I(z) \\
& =\lambda I_{0} \frac{[C(z) F(z)-z]\left[1-A^{*}\left(\lambda \alpha_{1}\right)\right]}{\lambda \alpha_{1}\left\{z-F(z)\left[A^{*}\left(\lambda \alpha_{1}\right)+C(z)\left(1-A^{*}\left(\lambda \alpha_{1}\right)\right)\right]\right\}} \\
& P_{i}(z) \\
& =\lambda I_{0} \frac{A^{*}\left(\lambda \alpha_{1}\right)[C(z)-1]\left[1-B_{i}^{*}\left(h_{i}(z)\right)\right] R_{i-1} X_{i-1}^{*}}{h_{i}(z)\left\{z-F(z)\left[A^{*}\left(\lambda \alpha_{1}\right)+C(z)\left(1-A^{*}\left(\lambda \alpha_{1}\right)\right)\right]\right\}} \\
& V_{i}(z) \\
& =\lambda I_{0} \frac{\tau_{i}\left[1-\theta_{i}-p_{i}(1-z)\right]\left[1-V_{i}^{*}\left(\lambda \alpha_{3}(1-C(z))\right)\right]}{\lambda \alpha_{3}(1-C(z))\left[1-B_{i}^{*}\left(h_{i}(z)\right)\right]} \\
& \left.D_{i}(z)=a_{i}(z)\right) h \\
& R_{i}(z) \\
& =a_{i} \frac{\left[1-D_{i}^{*}\left(\lambda \alpha_{4}(1-C(z))\right)\right]}{\lambda \alpha_{4}(1-C(z))} P_{i}(z) \\
& \lambda \alpha_{5}(1-C(z))
\end{aligned}
$$

Proof The partial generatingfunctions $I(z), P_{i}(z)$, $V_{i}(z), D_{i}(z)$ and $R_{i}(z)$ are defined as follow:

$$
\begin{aligned}
& I(z)=\int_{0}^{\infty} I(x, z) d x \\
& P_{i}(z)=\int_{0}^{\infty} P_{i}(x, z) d x,
\end{aligned}
$$

$D_{i}(z)=\int_{0}^{\infty} \int_{0}^{\infty} D_{i}(x, y, z) d x d y$.

$R_{i}(z)=\int_{0}^{\infty} \int_{0}^{\infty} R_{i}(x, y, z) d x d y$.

By substituting the equations (49)-(54) in these formulas, the relation in equation (58)-(62) can be obtained.

Also, to find $I_{0}$, the normalizing condition can be used 
$I_{0}$
$=\frac{1-F^{\prime}(1)-\left(1-A^{*}\left(\lambda \alpha_{1}\right)\right) C[1]}{1-F^{\prime}(1)+\lambda C[1] \sum_{i=1}^{k}\left\{R_{i-1} b_{i 1}\left[a_{i} g_{i 1}+a_{i} d_{i 1}-\tau_{i}\left(1-\theta_{i}\right) \frac{v_{i 1}}{b_{i 1}}-1\right]\right.}$.

\section{System performance measures}

In this section, some essential performance measures are derived. For this, the partial generating functions of the number of customers in the system and in orbit are introduced. Then by differentiating of these functions, the means of system and orbit size are obtained.

\section{Proposition}

a) The PGF of the number of customers in the system is

$\Omega(z)=I_{0} \frac{[C(z) F(z)-z]\left[1-A^{*}\left(\lambda \alpha_{1}\right)\right]+\alpha_{1}\left\{z-F(z)\left[A^{*}\left(\lambda \alpha_{1}\right)+C(z)\left(1-A^{*}\left(\lambda \alpha_{1}\right)\right)\right]+A^{*}\left(\lambda \alpha_{1}\right) W(z)\right\}}{\alpha_{1}\left\{z-F(z)\left[A^{*}\left(\lambda \alpha_{1}\right)+C(z)\left(1-A^{*}\left(\lambda \alpha_{1}\right)\right)\right]\right\}}$

where

$$
\begin{gathered}
W(z)=\sum_{i=1}^{k} \frac{R_{i-1} X_{i-1}^{*}}{\alpha_{3} \alpha_{4} \alpha_{5}}\left\{\alpha _ { 3 } [ 1 - B _ { i } ^ { * } ( h _ { i } ( z ) ) ] \left\{z \alpha_{4} \alpha_{5}+a_{i} \alpha_{5}\left[1-D_{i}^{*}\left(\lambda \alpha_{4}(1-C(z))\right)\right]\right.\right. \\
\left.+a_{i} \alpha_{4} D_{i}^{*}\left(\lambda \alpha_{4}(1-C(z))\right)\left[1-G_{i}^{*}\left(\lambda \alpha_{5}(1-C(z))\right)\right]\right\} \\
\left.+\alpha_{4} \alpha_{5} \tau_{i}\left[1-\theta_{i}-p_{i}(1-z)\right]\left[1-V_{i}^{*}\left(\lambda \alpha_{3}(1-C(z))\right)\right] B_{i}^{*}\left(h_{i}(z)\right) h_{i}(z)\right\}
\end{gathered}
$$

b) The PGF of the number of customers in orbit is

$\phi(\mathrm{z})=\mathrm{I}_{0} \frac{[\mathrm{C}(\mathrm{z}) \mathrm{F}(\mathrm{z})-\mathrm{z}]\left[1-\mathrm{A}^{*}\left(\lambda \alpha_{1}\right)\right]+\alpha_{1}\left\{\mathrm{z}-\mathrm{F}(\mathrm{z})\left[\mathrm{A}^{*}\left(\lambda \alpha_{1}\right)+\mathrm{C}(\mathrm{z})\left(1-\mathrm{A}^{*}\left(\lambda \alpha_{1}\right)\right)\right]+\mathrm{A}^{*}\left(\lambda \alpha_{1}\right) \mathrm{U}(\mathrm{z})\right\}}{\alpha_{1}\left\{\mathrm{z}-\mathrm{F}(\mathrm{z})\left[\mathrm{A}^{*}\left(\lambda \alpha_{1}\right)+\mathrm{C}(\mathrm{z})\left(1-\mathrm{A}^{*}\left(\lambda \alpha_{1}\right)\right)\right]\right\}}$

where

$$
\begin{gathered}
U(z)=\sum_{i=1}^{k} \frac{R_{i-1} X_{i-1}^{*}}{\alpha_{3} \alpha_{4} \alpha_{5}}\left\{\alpha _ { 3 } [ 1 - B _ { i } ^ { * } ( h _ { i } ( z ) ) ] \left\{\alpha_{4} \alpha_{5}+a_{i} \alpha_{5}\left[1-D_{i}^{*}\left(\lambda \alpha_{4}(1-C(z))\right)\right]\right.\right. \\
\left.+a_{i} \alpha_{4} D_{i}^{*}\left(\lambda \alpha_{4}(1-C(z))\right)\left[1-G_{i}^{*}\left(\lambda \alpha_{5}(1-C(z))\right)\right]\right\} \\
\left.+\alpha_{4} \alpha_{5} \tau_{i}\left[1-\theta_{i}-p_{i}(1-z)\right]\left[1-V_{i}^{*}\left(\lambda \alpha_{3}(1-C(z))\right)\right] B_{i}^{*}\left(h_{i}(z)\right) h_{i}(z)\right\}
\end{gathered}
$$

\section{Corollary}

a) The mean system size is:

$L_{s}=I_{0} \times \lim _{z \rightarrow 1} \frac{f^{/ /} g^{/}-g^{/ /} f^{/}}{2\left[g^{/}\right]^{2}}$

in which

$$
\begin{aligned}
& g(z)=\alpha_{1}\left\{z-F(z)\left[A^{*}\left(\lambda \alpha_{1}\right)+C(z)\left(1-A^{*}\left(\lambda \alpha_{1}\right)\right)\right]\right\} \\
& g^{\prime}(z)=\alpha_{1}\left\{1-F^{\prime}(z)\left[A^{*}\left(\lambda \alpha_{1}\right)+C(z)\left(1-A^{*}\left(\lambda \alpha_{1}\right)\right)\right]-C^{\prime}(z) F(z)\left(1-A^{*}\left(\lambda \alpha_{1}\right)\right)\right\} \\
& \lim _{z \rightarrow 1} g^{\prime}(z)=\alpha_{1}\left\{1-F^{\prime}(1)-C_{[1]}\left(1-A^{*}\left(\lambda \alpha_{1}\right)\right)\right\} \\
& g^{/ /}(z)=\alpha_{1}\left\{-F^{/ /}(z)\left[A^{*}\left(\lambda \alpha_{1}\right)+C(z)\left(1-A^{*}\left(\lambda \alpha_{1}\right)\right)\right]-2 C^{\prime}(z) F^{\prime}(z)\left(1-A^{*}\left(\lambda \alpha_{1}\right)\right)-C^{/ /}(z) F(z)\left(1-A^{*}\left(\lambda \alpha_{1}\right)\right)\right\} \\
& \lim _{z \rightarrow 1} g^{/ /}(z)=\alpha_{1}\left\{-F^{/ /}(1)-2 C_{[1]} F^{/}(1)\left(1-A^{*}\left(\lambda \alpha_{1}\right)\right)-C_{[2]}\left(1-A^{*}\left(\lambda \alpha_{1}\right)\right)\right\}
\end{aligned}
$$




$$
\begin{aligned}
& F(z)=\sum_{i=1}^{k}\left\{\left(1-\tau_{i}\right) p_{i} z+\left(1-\tau_{i}\right)\left(1-\theta_{i}-p_{i}\right)+\tau_{i}\left[1-\theta_{i}-p_{i}(1-z)\right]\right\} R_{i-1} X_{i}^{*} \\
& \lim _{z \rightarrow 1} F(z)=1 \\
& F^{\prime}(z)=\sum_{i=1}^{k}\left\{\left(1-\tau_{i}\right) p_{i}+\tau_{i} p_{i}\right\} R_{i-1} X_{i}^{*} \\
& +\sum_{i=1}^{k}\left\{\left(1-\tau_{i}\right) p_{i} z+\left(1-\tau_{i}\right)\left(1-\theta_{i}-p_{i}\right)+\tau_{i}\left[1-\theta_{i}-p_{i}(1-z)\right]\right\} R_{i-1} \frac{d}{d z} X_{i-1}^{*} \\
& \lim _{z \rightarrow 1} \frac{d}{d z} X_{i}^{*}=\sum_{j=1}^{i} h_{j}^{\prime}(1) b_{j 1} \\
& h_{i}(z)=\lambda \alpha_{2}(1-C(z))+a_{i}\left[1-D_{i}^{*}\left(\lambda \alpha_{4}(1-C(z))\right)\right] G_{i}^{*}\left(\lambda \alpha_{5}(1-C(z))\right) \\
& h_{i}^{\prime}(z)=-\lambda \alpha_{2} C^{\prime}(z)+a_{i} \lambda \alpha_{4} C^{\prime}(z) \frac{d}{d z} D_{i}^{*}\left(\lambda \alpha_{4}(1-C(z))\right) G_{i}^{*}\left(\lambda \alpha_{5}(1-C(z))\right) \\
& -a_{i} \lambda \alpha_{5} C^{\prime}(z) D_{i}^{*}\left(\lambda \alpha_{4}(1-C(z))\right) \frac{d}{d z} G_{i}^{*}\left(\lambda \alpha_{5}(1-C(z))\right) \\
& h_{i}^{\prime}(1)=\lim _{z \rightarrow 1} \frac{d}{d z} h_{i}^{\prime}(z) \\
& h_{i}^{\prime}(1)=-\lambda C_{[1]}\left\{\alpha_{2}+a_{i}\left(\alpha_{4} d_{i 1}+\alpha_{5} g_{i 1}\right)\right\} \\
& \overline{h_{i}}(1)=\lim _{z \rightarrow 1} \frac{d^{2}}{d z^{2}} h_{i}(z)=\sum_{i=1}^{k}\left(R_{i-1}-R_{i}\right) \frac{d}{d z} X_{i-1}^{*} \\
& =\sum_{i=1}^{k} \sum_{j=1}^{i-1}\left(R_{i-1}-R_{i}\right) \lambda C_{[1]}\left\{\alpha_{2}+a_{i}\left(\alpha_{4} d_{i 1}+\alpha_{5} g_{i 1}\right)\right\} b_{j 1} \\
& \lim _{z \rightarrow 1} F^{\prime}(z)=\sum_{i=1}^{k} p_{i} R_{i-1}+\sum_{i=1}^{k} \sum_{j=1}^{i-1}\left(R_{i-1}-R_{i}\right) \lambda C_{[1]}\left\{\alpha_{2}+a_{i}\left(\alpha_{4} d_{i 1}+\alpha_{5} g_{i 1}\right)\right\} b_{j 1} \\
& \lim _{z \rightarrow 1} F^{/ /}(z)=2 \sum_{i=1}^{k} p_{i} R_{i-1} \sum_{j=1}^{i} b_{j 1} h_{j}^{\prime}(1)+\sum_{i=1}^{k} \sum_{j=1}^{i-1}\left(R_{i-1}-R_{i}\right)\left[b_{j 1} h_{j}^{/ /}(1)+b_{j 2}\left(h_{j}^{\prime}(1)\right)^{2}\right] \\
& h_{j}^{\prime /}(1)=-C_{[2]} \lambda \alpha_{2}-C_{[2]} \lambda a_{i}\left(\alpha_{4} d_{i 1}+\alpha_{5} g_{i 1}\right)+C_{[1]}^{2} \lambda^{2} a_{i}\left(\alpha_{4}{ }^{2} d_{i 2}+\alpha_{5}^{2} g_{i 2}+2 \alpha_{4} \alpha_{5} d_{i 1} g_{i 1}\right) \\
& f(z)=[C(z) F(z)-z]\left[1-A^{*}\left(\lambda \alpha_{1}\right)\right]+\alpha_{1}\left\{z-F(z)\left[A^{*}\left(\lambda \alpha_{1}\right)+C(z)\left(1-A^{*}\left(\lambda \alpha_{1}\right)\right)\right]+A^{*}\left(\lambda \alpha_{1}\right) W(z)\right\} \\
& f^{\prime}(z)=\left\{C^{\prime}(z) F(z)+C(z) F^{\prime}(z)-1\right\}\left(1-A^{*}\left(\lambda \alpha_{1}\right)\right) \\
& +\alpha_{1}\left\{1-F^{\prime}(z)\left[A^{*}\left(\lambda \alpha_{1}\right)+C(z)\left(1-A^{*}\left(\lambda \alpha_{1}\right)\right)\right]-C^{\prime}(z) F(z)\left(1-A^{*}\left(\lambda \alpha_{1}\right)\right)+A^{*}\left(\lambda \alpha_{1}\right) W^{\prime}(z)\right\} \\
& f^{\prime}(1)=\lim _{z \rightarrow 1} f^{\prime}(z)=\left[C_{[1]}+F^{\prime}(1)-1\right]\left(1-A^{*}\left(\lambda \alpha_{1}\right)\right)+\alpha_{1}\left\{1-F^{\prime}(1)-C_{[1]}\left(1-A^{*}\left(\lambda \alpha_{1}\right)\right)+A^{*}\left(\lambda \alpha_{1}\right) W^{\prime}(1)\right\} \\
& f^{\prime /}(z)=\left\{C^{/ /}(z) F(z)+2 C^{\prime}(z) F^{\prime}(z)+C(z) F^{/ /}(z)\right\}\left(1-A^{*}\left(\lambda \alpha_{1}\right)\right) \\
& +\alpha_{1}\left\{F^{/ /}(z)\left[A^{*}\left(\lambda \alpha_{1}\right)+C(z)\left(1-A^{*}\left(\lambda \alpha_{1}\right)\right)\right]-2 C^{\prime}(z) F^{\prime}(z)\left(1-A^{*}\left(\lambda \alpha_{1}\right)\right)\right. \\
& \left.-F(z) C^{/ /}(z)\left(1-A^{*}\left(\lambda \alpha_{1}\right)\right)+A^{*}\left(\lambda \alpha_{1}\right) W^{/ /}(z)\right\} \\
& f^{/ /}(1)=\lim _{z \rightarrow 1} f^{/ /}(z)=\left\{\left(1-\alpha_{1}\right)\left[C_{[2]}+2 F^{\prime}(1) C_{[1]}\right]+F^{/ /}(1)\right\}\left(1-A^{*}\left(\lambda \alpha_{1}\right)\right)+\alpha_{1}\left[W^{/ /}(1) A^{*}\left(\lambda \alpha_{1}\right)-F^{/ /}(1)\right]
\end{aligned}
$$




$$
\begin{aligned}
W(z)=\sum_{i=1}^{k} \frac{R_{i-1} X_{i-1}^{*}}{\alpha_{3} \alpha_{4} \alpha_{5}}\left\{\alpha _ { 3 } [ 1 - B _ { i } ^ { * } ( h _ { i } ( z ) ) ] \left\{z \alpha_{4} \alpha_{5}+a_{i} \alpha_{5}\left[1-D_{i}^{*}\left(\lambda \alpha_{4}(1-C(z))\right)\right]\right.\right. \\
\left.+a_{i} \alpha_{4} D_{i}^{*}\left(\lambda \alpha_{4}(1-C(z))\right)\left[1-G_{i}^{*}\left(\lambda \alpha_{5}(1-C(z))\right)\right]\right\} \\
\left.+\alpha_{4} \alpha_{5} \tau_{i}\left[1-\theta_{i}-p_{i}(1-z)\right]\left[1-V_{i}^{*}\left(\lambda \alpha_{3}(1-C(z))\right)\right] B_{i}^{*}\left(h_{i}(z)\right) h_{i}(z)\right\}
\end{aligned}
$$

$W^{\prime}(z)=\frac{d W(z)}{d z}$

$$
W^{\prime}(1)=\lim _{z \rightarrow 1} W^{\prime}(z)=-\sum_{i=1}^{k} R_{i-1}\left[h_{i}^{\prime}(1) b_{i 1}+\frac{\tau_{i}\left(1-\theta_{i}\right)}{\alpha_{3}} v_{i 1}\right]
$$

$W^{/ /}(1)=\lim _{z \rightarrow 1} W^{/ /}(z)$

$$
\begin{aligned}
& =-2 \sum_{i=1}^{k} \frac{d X_{i-1}^{*}}{d z} R_{i-1}\left[h_{i}^{\prime}(1) b_{i 1}-\frac{\tau_{i}\left(1-\theta_{i}\right)}{\alpha_{3}} v_{i 1}\right] \\
& +\sum_{i=1}^{k} \frac{R_{i-1}}{\alpha_{3} \alpha_{4} \alpha_{5}}\left\{-\alpha_{3} \alpha_{4} \alpha_{5}\left[h_{i}^{\prime \prime}(1) b_{i 1}+\left(h_{i}^{\prime}(1)\right)^{2} b_{i 2}-2 h_{i}^{\prime}(1) b_{i 1}\right]\right\}+2 \alpha_{3} h_{i}^{\prime}(1) a_{i} b_{i 1}\left(\alpha_{5} d_{i 1}+\alpha_{4} g_{i 1}\right) \\
& -2 \alpha_{4} \alpha_{5} \tau_{i} v_{i 1}\left\{p_{i}+\left(1-\theta_{i}\right)\left[h_{i}^{\prime}(1)+b_{i 1}\right]\right\}-\alpha_{4} \alpha_{5} \tau_{i} v_{i 2}\left(1-\theta_{i}\right)
\end{aligned}
$$

b)

The mean orbit size is:

$$
L_{q}=I_{0} \times \lim _{z \rightarrow 1} \frac{m^{/ /}(z) g^{/}(z)-g^{/ /}(z) m^{\prime}(z)}{2\left[g^{/}(z)\right]^{2}}
$$

in which

$$
\begin{aligned}
& m(z)=[C(z) F(z)-z]\left[1-A^{*}\left(\lambda \alpha_{1}\right)\right]+\alpha_{1}\left\{z-F(z)\left[A^{*}\left(\lambda \alpha_{1}\right)+C(z)\left(1-A^{*}\left(\lambda \alpha_{1}\right)\right)\right]+A^{*}\left(\lambda \alpha_{1}\right) U(z)\right\} \\
& m^{\prime}(z)=\left\{C^{\prime}(z) F(z)+C(z) F^{\prime}(z)-1\right\}\left(1-A^{*}\left(\lambda \alpha_{1}\right)\right) \\
& +\alpha_{1}\left\{1-F^{\prime}(z)\left[A^{*}\left(\lambda \alpha_{1}\right)+C(z)\left(1-A^{*}\left(\lambda \alpha_{1}\right)\right)\right]-C^{\prime}(z) F(z)\left(1-A^{*}\left(\lambda \alpha_{1}\right)\right)+A^{*}\left(\lambda \alpha_{1}\right) U^{\prime}(z)\right\} \\
& m^{\prime}(1)=\lim _{z \rightarrow 1} m^{\prime}(z)=\left[C_{[1]}+F^{\prime}(1)-1\right]\left(1-A^{*}\left(\lambda \alpha_{1}\right)\right)+\alpha_{1}\left\{1-F^{\prime}(1)-C_{[1]}\left(1-A^{*}\left(\lambda \alpha_{1}\right)\right)+A^{*}\left(\lambda \alpha_{1}\right) U^{\prime}(1)\right\} \\
& m^{/ /}(z)=\left\{C^{/ /}(z) F(z)+2 C^{/}(z) F^{/}(z)+C(z) F^{/ /}(z)\right\}\left(1-A^{*}\left(\lambda \alpha_{1}\right)\right) \\
& +\alpha_{1}\left\{F^{/ /}(z)\left[A^{*}\left(\lambda \alpha_{1}\right)+C(z)\left(1-A^{*}\left(\lambda \alpha_{1}\right)\right)\right]-2 C^{\prime}(z) F^{\prime}(z)\left(1-A^{*}\left(\lambda \alpha_{1}\right)\right)\right. \\
& \left.-F(z) C^{/ /}(z)\left(1-A^{*}\left(\lambda \alpha_{1}\right)\right)+A^{*}\left(\lambda \alpha_{1}\right) U^{/ /}(z)\right\} \\
& m^{/ /}(1)=\lim _{z \rightarrow 1} m^{/ /}(z)=\left\{\left(1-\alpha_{1}\right)\left[C_{[2]}+2 F^{\prime}(1) C_{[1]}\right]+F^{/ /}(1)\right\}\left(1-A^{*}\left(\lambda \alpha_{1}\right)\right)+\alpha_{1}\left[U^{/ /}(z) A^{*}\left(\lambda \alpha_{1}\right)-F^{/ /}(1)\right] \\
& U^{\prime}(z)=\frac{d U(z)}{d z} \\
& U^{\prime}(1)=\lim _{z \rightarrow 1} U^{\prime}(z)=-\sum_{i=1}^{k} R_{i-1}\left[h_{i}^{\prime}(1) b_{i 1}+\frac{\tau_{i}\left(1-\theta_{i}\right)}{\alpha_{3}} v_{i 1}\right] \\
& U^{/ /}(1)=\lim _{z \rightarrow 1} U^{/ /}(z) \\
& =-2 \sum_{i=1}^{k} \frac{d X_{i-1}^{*}}{d z} R_{i-1}\left[h_{i}^{\prime}(1) b_{i 1}+\frac{\tau_{i}\left(1-\theta_{i}\right)}{\alpha_{3}} v_{i 1}\right] \\
& +\sum_{i=1}^{k} \frac{R_{i-1}}{\alpha_{3} \alpha_{4} \alpha_{5}}\left\{-\alpha_{3} \alpha_{4} \alpha_{5}\left[h_{i}^{\prime \prime}(1) b_{i 1}+\left(h_{i}^{\prime}(1)\right)^{2} b_{i 2}\right]\right\}+2 \alpha_{3} h_{i}^{\prime}(1) a_{i} b_{i 1}\left(\alpha_{5} d_{i 1}+\alpha_{4} g_{i 1}\right) \\
& -2 \alpha_{4} \alpha_{5} \tau_{i} v_{i 1}\left\{p_{i}+\left(1-\theta_{i}\right)\left[h_{i}^{\prime}(1)+b_{i 1}\right]\right\}-\alpha_{4} \alpha_{5} \tau_{i} v_{i 2}\left(1-\theta_{i}\right)
\end{aligned}
$$




\section{Proof of corollary}

a) By differentiating of the $\Omega(z)$ in (69),taking limit at $z=1$ and applying L'Hopital rule we have

$$
L_{s}=\lim _{z \rightarrow 1} d \Omega(z) / d z \text {. }
$$

b) By differentiating of the $\phi(z)$ in (70), taking limit at $z=1$ and applying L'hopital rule we have

$$
L_{q}=\lim _{z \rightarrow 1} d \phi(z) / d z
$$

In follow, we can obtain the mean of waiting time of customers at the system and orbit by using Little's formula and relations (71) and (72) as follows:

$$
W_{s}=L_{s} / \lambda, \text { and } W_{q}=L_{q} / \lambda .
$$

\section{The Numerical Example (The inspection test of valves)}

In this section, the results of a numerical example are shown in a specific case of this model.

The inspection process of a valve producer can be considered as an application of this model. The valves are used at several engineering systems, thus their quality and inspection processes are very important for them. This process can be done in 3 phases (Table 1). So, the valves are sent to a station individually with Poisson distribution with rate $\lambda$ per hour. It is possible to inspect all the valves $\left(\alpha_{i}=1, i=1,2,3,4,5\right)$. Also the retrial times in this process have an exponential distribution with rate $\eta$ per hour. The vacation is occurred at the end of third phase and the vacation times are distributed exponential with mean $v_{3}$ (min).In this example, the probability of failure is considered zero, so the effect of delay and repair variables is not examined.

Table 1.The values of the parameters of model for quality control of valves

\begin{tabular}{|c|l|c|c|c|c|c|c|c|c|}
\hline $\begin{array}{c}\text { Number of } \\
\text { phases } \\
(i)\end{array}$ & Name of phases & $\begin{array}{c}\text { Distribution } \\
\text { of service } \\
\text { times }\end{array}$ & $\begin{array}{c}\text { Mean of } \\
\text { service times } \\
(\mathrm{min})\end{array}$ & $\begin{array}{c}\text { The probability of pass of the phase } \\
\left(\theta_{\mathrm{i}}, i=1,2 \text { and } q_{3}\right. \\
\text { (Three cases are considered for } \\
\text { different value of reliability) }\end{array}$ & \multicolumn{2}{|c|}{$p_{i}$} \\
\hline 1 & Painting inspection & $\begin{array}{c}\text { Exponential( } \\
\text { Exp) }\end{array}$ & 3 & 0.999999 & 0.8 & 0.65 & \multicolumn{2}{|c|}{0.1} & 0 \\
\hline 2 & Pressure tests & Exp & 5 & 0.999999 & 0.8 & 0.65 & \multicolumn{2}{|c|}{0.1} & 0 \\
\hline 3 & $\begin{array}{l}\text { Operation } \\
\text { inspection manual } \\
\text { operating type }\end{array}$ & Exp & 10 & 0.999999 & 0.8 & 0.65 & $1-10^{-6}$ & 0.2 & 0.35 \\
\hline
\end{tabular}

Then, the results of the sensitivity analysis of the test inspection respect to the values of $\lambda, \eta$ and $v_{3}$ at different values of reliability in the Fig. 1., Fig. 2. and Fig. 3., in the following:

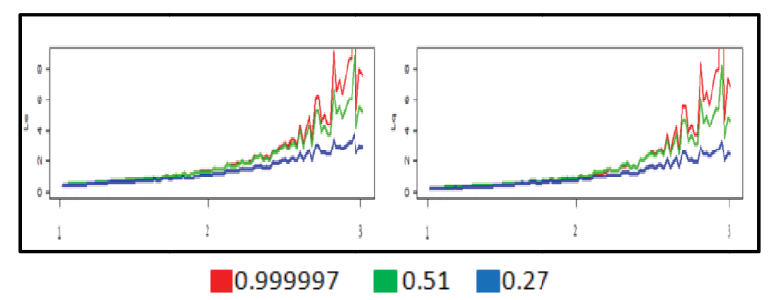

Figure 1. Performance measures for various values of $\lambda([1,3])$ and RE when $\eta=10$ and $v_{3}=6$

As Figure 1 shows, $L_{s}$ and $L_{q}$ experience an increasing trend, when $\lambda$ tends to increase.
Figure 2 demonstrates that, $L_{s}$ and $L_{q}$ experience a decreasing trend, when $\eta$ increases.

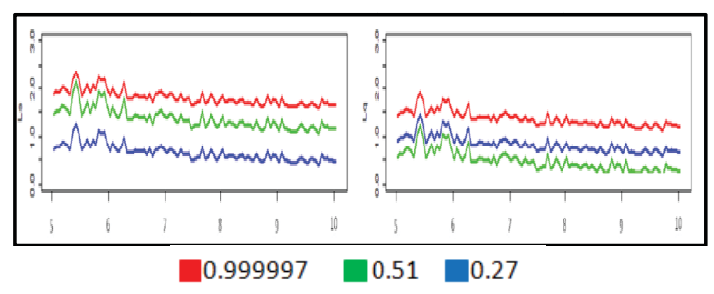

Figure 2. Performance measures for various values of $\eta$ $([5,10])$ and RE when $\lambda=2$ and $v_{3}=6$ 


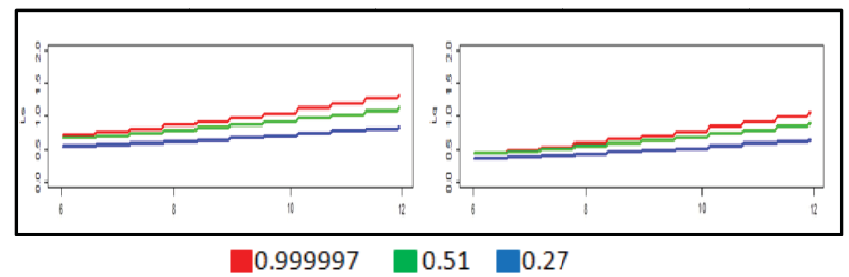

Figure 3. Performance measures for various values of $\boldsymbol{v}_{3}$ $([6,12])$ and $\mathrm{RE}$ when $\lambda=2$ and $\eta=10$

It seems from Figure 3 that, $L_{s}$ and $L_{q}$ experience an increasing trend when $v$ tends to increase.

Also, Figure 1, 2 and 3 show that, by increasing the value of reliability, the $L_{s}$ and $L_{q}$ are increased.

Thus, the $r_{i}$ and $q_{3}$ probabilities $(i=1,2)$, should be increased to reliability increasing. Consequently, the following comments can help to increase reliability.

(1) The casting process output has to be tested.

(2) Raw materials used for stem valves manufacturing, should be evaluated and controlled.

(3) According to the experiment and experts comment, all fastens and connections should be checked by standard gauges. This task improves flanges quality and reduces the rejection rate.

(4) Checking punches of valves after assembly by using Bench test

\section{Conclusion}

In thispaper, $\mathrm{a} M^{X} / G / 1$ retrial queueing model with first essential phase and $\mathrm{k}-1$ optional phases of service, probabilistic feedback, failure, delay, repair, and vacation conditions at each phase is considered. For this model, the steady-state equations, the generating functions of the number of customers in the system and orbit, and some important performance measures such as the mean of the system and orbit size and the mean of the system and orbit waiting times have been derived by the supplementary variable technique. Then, in a specificcase of this model, the sensitivity analysis of performance measures via model parameters in different values of the reliability is conducted.According to the results of this analysis, some technical suggestions are presentedfor managing the parameters of the model in order to control and optimize the performance of the system. In the future researches, the modeling and analyzing multi-stage systems with several servers and the systems which their service phases times are dependent can be considered.

\section{References}

[1] John, F.Sh., James, M.Th.,Donald, G.,Carl, M.H.: Fundamentals of Queueing Theory. Wiley Series in Probability and Statistics (2018)
[2] Falin, G.I., Templeton, J.G.C.: Retrial queues. London, Chapman and Hall (1997)

[3] Artalejo, J.R.: Accessible bibliography on retrial queues. Math. Comput. Model. 30, 1-6 (1999)

[4] Falin, G.I.: On a multiclass batch arrival retrial queue. Adv. Appl. Probab. 20, 483-487 (1988)

[5] Kulkarni, V.G.: Expected waiting times in a multiclass batch arrival retrial queue. J. Appl. Probab. 23, 144-154 (1986)

[6] Yamamuro, K. The queue length in an M/G/1 batch arrival retrial queue. QueueingSyst 70, 187-205 (2012)

[7] Kumar, B.K., Kumar, A.V., Arivudainambi, D.: An $\mathrm{M} / \mathrm{G} / 1$ retrial queueing system with two phase service and preemptive resume. Ann. Oper. Res. 113, 61-79 (2002)

[8] Choudhury, G., Deka, K.: An $M / G / 1$ retrial queueing system with two phases of service subject to the server breakdown and repair. Perform. Evaluation. 65(10), 714-724 (2008)

[9] Wang, J., Li, J.: A single server retrial queue with general retrial times and two phase service. J. Syst. Sci. Complex. 22, 291-302 (2009)

[10] Maurya, V.N.: Sensitivity analysis on significant performance measures of bulk arrival retrial queueing $M^{X} /\left(G_{1}, G_{2}\right) / 1$ model with second phase optional service and Bernoulli vacation schedule. Int. Open. J.Oper. Res. 1(1), 1 - 15 (2013)

[11] Jeganathan, K., Kathiresan, J., Anbazhagan, N.: A retrial inventory system with priority customers and second optional service. Opsearch. 53, 808-834 (2016)

[12] Rao, S.H., Vemuri, V.K., Kumar B.S., Rao T.S.: Analysis of two-Phase queueing system with impatient customers, server breakdowns and delayed repair. Int. J. Pure. Appl. Math. 115(4), 651-663 (2017)

[13] Choudhury, G., Paul, M.: A two phase queueing system with Bernoulli feedback. Inf. Manag. Sci. 16(1), 35-52 (2005)

[14] Arivudainambi, D., Godhandaraman, P.: A batch arrival retrial queue with two phases of service, feedback and $\mathrm{K}$ optional vacations. Appl. Math. Sci. 6(22), 1071-1087 (2012)

[15] BadamchiZadeh, A.: A batch arrival multiphase queueing system with random feedback in service and single vacation policy. Opsearch. 52(4), 617-630 (2015)

[16] Som, B.K., Seth, S.: M/M/c/N queueing systems with encouraged arrivals, reneging, retention and feedback customers. Yugosl. J. Oper. Res. 28(00), 6-6 (2018)

[17] Rajadurai, P., Chandrasekaran, V.M., Saravanarajan, M.C.: Analysis of an $M^{X} / G / 1$ unreliable retrial G-queue with orbital search 
and feedback under Bernoulli vacation schedule. Opsearch. 53(1), 197-223 (2016)

[18] Bouchentouf, A.A., Cherfaoui, M., Boualem, M.: Performance and economic analysis of a single server feedback queueing model with vacation and impatient customers. Opsearch. 56, 300-323 (2019)

[19] Senthikumar, R.,Arumuganathan,M.: On the single server batch arrival queue with general vacation time under Bernoulli schedule and two phases of heterogenous service. Quality Technology and Quantitative Management. 5, 145-160 (2008)

[20] Jain, M., Bhagat, A.: $M^{X} / G / 1$ retrial vacation queue for multi-optional services, phase repair and reneging. Quality Technology and Quantitative Management. 13(3), 63-288 (2016)

[21] Choudhury, G., Ke,JC.: A batch arrival retrial queue with general retrial times under bernoulli vacation schedule for unreliable server and delaying repair. Applied Mathemathical Modeling. 36, 255-269 (2012)

[22] Azhagappan, A.: Transient behavior of a Markovianqueue with working vacation variant reneging and awaiting server. TOP 27,351 (2019)

[23] Ke,JC.: Operating characteristic analysis on the $\mathrm{MX} / \mathrm{G} / 1$ system with a variant vacation policy and balking. Applied Mathematical Modeling. 31,1321-1337 (2007)

[24] Kulkarni, V.G.,Bong, D. C.: Retrial queues with server subject to breakdowns and repairs. Queueing Systems.7, 191-208(1990)

[25] Rajadurai, P., Saravanarajan, M.C., Chandrasekaran, V.M.: A study on $\mathrm{M} / \mathrm{G} / 1$ feedback retrial queue with subject to server breakdown and repair under multiple working vacation policy.Alexandria Engineering Journal.57(2), 947-962 (2018)

[26] Jain, M.,Bhagat, A.: Unreliable bulk retrial queues with delayed repairs and modified vacation policy.Journal of Industrial Engineering International. 10, Article number: 63 (2014)

[27] Choudhury, G., Deka, K.: An $M^{X} / G / 1$ unreliable retrial queue with two phases of service and Bernoulli admission mechanism. Appl. Math. Comput. 215(3), 936-949 (2009)

[28] Choudhury, G., Deka, K.: A batch arrival retrial queueing system with two phases of service and service interruption. Comput. Math. with Appl. 59(1), 437-450 (2010)

[29] Birnbaun,ZW.,Esary, JD., Saunders, SD.: Multi-component systems and structures and their reliability. Technometrics. 3(1): 55-77 (1961)

[30] Li, W., Shi, D., Chao, X.: Reliability analysis of $\mathrm{M} / \mathrm{G} / 1$ queueing system with server breakdowns and vacations. Journal of Applied Probability. 34: 546-555 (1997)

[31] Tang, Y.: A Single Server M/G/1 Queueing system subject to breakdowns: some reliability and queueing problems. Microelectronics Reliability. 37: 315-321 (1997)

[32] Wang, J., Cao, J., Li, Q.: Reliability analysis of the retrial queue with server breakdowns and repairs. Queueing Systems 38: 363-380. (2001)

[33] Achcar,JA.,Piratelli, CL.: Modeling quality control data Weibull distributions in the presence of a change point. The International Journal of Advanced Manufacturing Technology. 66: 1611-1621 (2013)

[34] Dept. of Def. of USA.: Electronic reliability design handbook. MIL-HDBK-338B, USA (1998)

[35] Dept. of Def. of USA.: Unmanned aerial vehicle reliability study. Office of the secretary of defence, USA (2003)

[36] Dept. of NAVY of USA (NSWC).: Handbook of reliability prediction procedures for mechanical equipment. Bethesda, Maryland, 20817-5700,USA2006). 